\title{
Local recurrence and subsequent endoscopic treatment after endoscopic piecemeal mucosal resection with or without precutting in the colorectum
}

\author{
Myeongsook Seo, Eun Mi Song, Gwang Un Kim, Sung Wook Hwang, Sang Hyoung Park, Dong-Hoon Yang, \\ Kyung-Jo Kim, Byong Duk Ye, Seung-Jae Myung, Suk-Kyun Yang, Jeong-Sik Byeon \\ Department of Gastroenterology, Asan Medical Center, University of Ulsan College of Medicine, Seoul, Korea
}

\begin{abstract}
Background/Aims: Precutting before endoscopic piecemeal mucosal resection (EPMR) may increase colorectal polyp resection effectiveness. We aimed to identify risk factors for recurrence after conventional EPMR (CEPMR) and precut EPMR (PEPMR) and investigated endoscopic treatment outcomes for recurrent cases. Methods: The medical records of patients with colorectal polyps treated by EPMR were analyzed. Patients without follow-up surveillance colonoscopies were excluded. Results: Among 359 lesions, the local recurrence rate on the first surveillance colonoscopy was 5.8\% (18/312) and 6.4\% (3/47) after CEPMR and PEPMR, respectively. Among lesions without recurrence at the first surveillance colonoscopy, the rates of late recurrence on subsequent surveillance colonoscopy were 3.9\% (6/152) and 0\% after CEPMR and PEPMR, respectively. Larger tumor size was the only independent risk factor for recurrence (odds ratio, 7.93; 95\% confidence interval, $1.95-32.30 ; P<0.001$ ). Endoscopic treatment was performed for all 27 recurrences. A combination of $\geq 2$ endoscopic treatment modalities was used in 19 of 27 recurrences (70.4\%). Surveillance colonoscopies were performed in 20 of 27 recurrences after endoscopic treatment. One (5.0\%) had a re-recurrence and was treated by surgical resection because recurrence occurred at the appendiceal orifice. Nineteen of 20 lesions (95.0\%) could be cured endoscopically, although 3 of the 19 showed second or third recurrences and were treated by repeat endoscopic resection. Conclusions: The local recurrence rates after CEPMR and PEPMR were similar. Larger tumor size was an independent risk factor for local recurrence after EPMR. Endoscopic treatment of recurrences resulted in high cure rates, although combination methods were necessary in many cases. (Intest Res 2017;15:502-510)
\end{abstract}

Key Words: Endoscopic mucosal resection; Colonic neoplasia; Piecemeal resection

\section{INTRODUCTION}

The endoscopic mucosal resection (EMR) technique has been widely used for the resection of colorectal neoplasms. Endoscopic piecemeal mucosal resection (EPMR) is used in up to $50 \%$ of cases for resection of large lesions of $>20 \mathrm{~mm}$ in diameter. ${ }^{1-4}$ The local recurrence rate is higher with EPMR

Received December 28, 2016. Revised March 20, 2017. Accepted March 29, 2017. Published online July 19, 2017

Correspondence to Jeong-Sik Byeon, Department of Gastroenterology, Asan Medical Center, University of Ulsan College of Medicine, 88 Olympic-ro 43-gil, Songpa-gu, Seoul 05505, Korea. Tel: +82-2-3010-3905, Fax: +82-23010-5782,E-mail: jsbyeon@amc.seoul.kr

Financial support: None. Conflict of interest: None. than with en bloc resection. The local recurrence rate after EPMR has been reported to be up to $50 \%$, compared with a rate of $0 \%$ to $17.8 \%$ after en bloc resection. ${ }^{1,5,6}$ Endoscopic submucosal dissection is an effective procedure for en bloc resection of large colorectal neoplasms. However, endoscopic submucosal dissection has not been widely used because it is technically difficult to perform and carries a considerable risk of perforation. ${ }^{5,7-10}$ Therefore, EMR with circumferential precutting (precut EMR) has been introduced as an alternative method for resecting large polyps. ${ }^{11}$ Several studies demonstrated that precut EMR had higher en bloc resection rates and lower recurrence rates than conventional EMR. ${ }^{8,12}$ However, precut EMR also had a piecemeal resection rate

\footnotetext{
(c) Copyright 2017. Korean Association for the Study of Intestinal Diseases. All rights reserved.

This is an Open Access article distributed under the terms of the Creative Commons Attribution Non-Commercial License (http://creativecommons.org/licenses/by-nc/4.0)

which permits unrestricted non-commercial use, distribution, and reproduction in any medium, provided the original work is properly cited.
} 
with a range of $34.0 \%$ to $54.9 \%$ in large lesions. ${ }^{8,12}$ Despite this high piecemeal rate with precut EMR, the recurrence rate after precut EPMR (PEPMR) has not been extensively investigated compared with conventional EPMR (CEPMR).

There have been few studies of the clinical outcome of endoscopic treatment for recurrent lesions after EPMR. ${ }^{1,13,14} \mathrm{~A}$ recent Australian study of colonic EMR reported that 135 of 145 recurrent/residual adenomas (93.1\%) were successfully treated endoscopically. ${ }^{1}$ However, this study did not describe the detailed methods, such as EMR, avulsion using hot biopsy forceps, and argon plasma coagulation (APC). Thus, further studies are necessary to evaluate the performance and outcome of salvage treatment for recurrent lesions after EPMR.

The aims of our study were to compare the outcomes of CEPMR and PEPMR and to identify risk factors for local recurrence after EPMR. In addition, we aimed to investigate the performance and outcome of endoscopic treatment for recurrent lesions.

\section{METHODS}

\section{Patients}

We retrospectively analyzed data regarding 359 lesions in
344 patients who underwent EPMR and who had at least 1 surveillance colonoscopy or sigmoidoscopy after the initial EPMR for a colorectal polyp of $\geq 10 \mathrm{~mm}$ diameter at the Asan Medical Center between January 2005 and December 2014 (Fig. 1). We searched for EPMR cases by searching for the term "piecemeal" in the colonoscopy report found with a search of the computerized database at our institution. After initial selection of EPMR cases by this system, we reviewed all the colonoscopy images of these patients and finally enrolled the EPMR cases in this study. We excluded patients with a positive deep resection margin. All procedures were performed by 8 experienced endoscopists and 15 trainee endoscopists using CEPMR or PEPMR methods. All of the 8 experienced endoscopists had $>10$ years of experience in diagnostic and therapeutic colonoscopies. They performed 94.9\% of the CEPMR cases and $95.7 \%$ of the PEPMR cases. Cases in which the patient had undergone EPMR for subepithelial lesions, such as neuroendocrine tumor, leiomyoma, and granular cell tumor were excluded; the patients who had familial adenomatous polyposis or UC were also excluded. All patients provided written informed consent to undergo endoscopic resection of colorectal polyps. This study was approved by the Institutional Review Board of the Asan Medical Center, Seoul, Korea (IRB number: 2015-0954) and

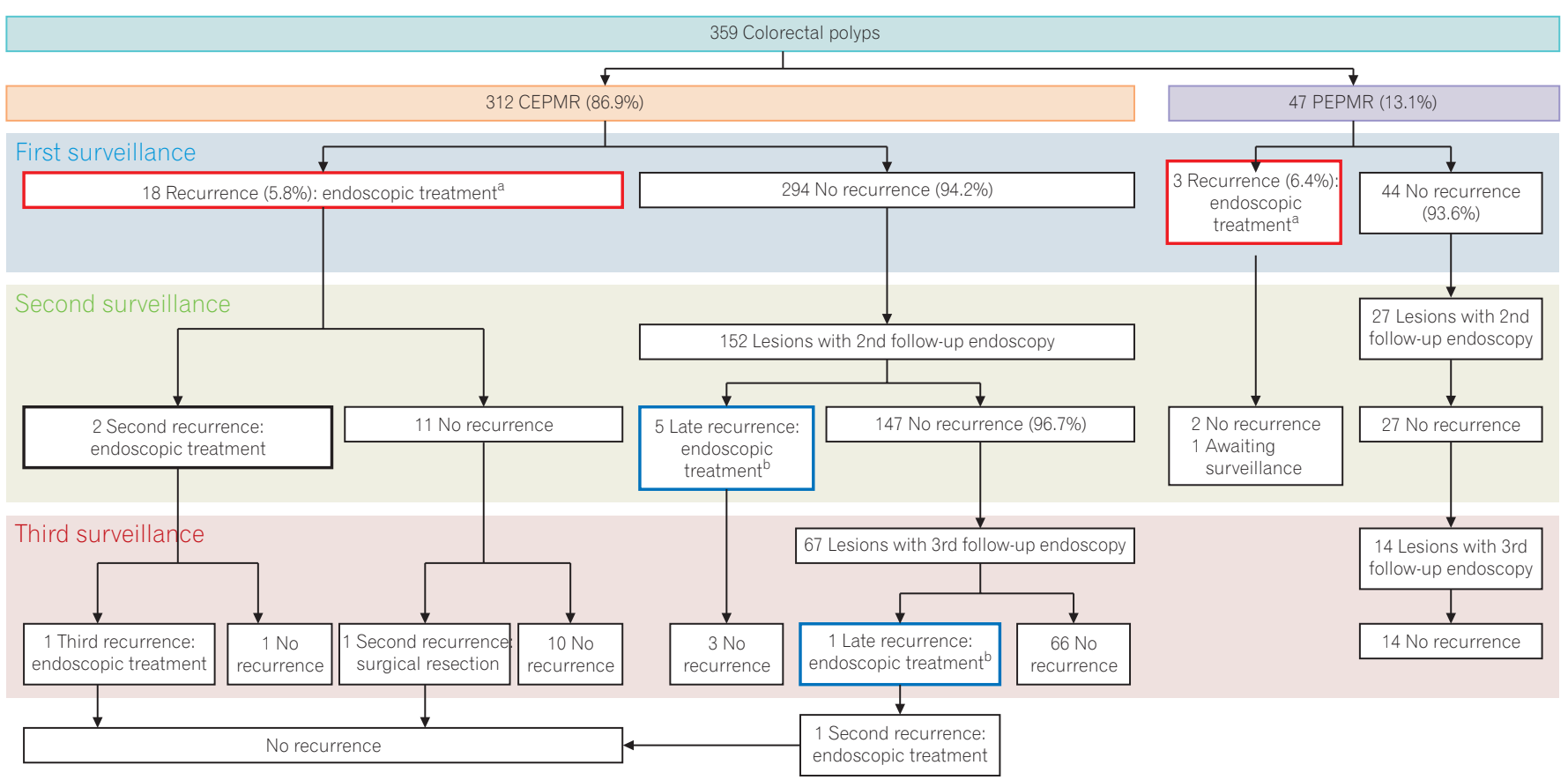

Fig. 1. Flow diagram showing recurrence and subsequent endoscopic treatment. ${ }^{a}$ Early recurrence (red box) rates at the first surveillance were $5.8 \%$ (18/312) and 6.4\% (3/47) after conventional endoscopic piecemeal mucosal resection (CEPMR) and precut EPMR (PEPMR), respectively; ${ }^{b}$ Among lesions without recurrence at the first surveillance colonoscopy, the rates of late recurrence (blue box) were 3.9\% (6/152) and 0\% after CEPMR and PEPMR, respectively. In summary, the overall recurrence rates were 7.7\% (24/312) and 6.4\% (3/47) after CEPMR and PEPMR, respectively. 
waived for informed consent.

\section{EPMR Procedures}

A single-channel endoscope was used to perform EPMR procedures. We used a mixed solution of normal saline with epinephrine $(1: 10,000)$ for submucosal injection. A small amount of indigo carmine was added to the solution to improve the visibility of the submucosal layer and polyp edges. For CEPMR, after submucosal injection, part of the polyp was snared and resected by electrical current. Repeat snaring and resection was performed until most of the lesion had been resected. If a small residual lesion was left, avulsion was performed with the use of hot biopsy forceps. If a tiny invisible residual was suspected because of multiple resections, APC or snare tip coagulation was used for fulguration. For PEPMR, a circumferential incision was made 2 to $4 \mathrm{~mm}$ from the edge of the polyp by electrical cutting with the snare tip or dual knife (Olympus Co., Tokyo, Japan) after submucosal injection. A snare was placed at the cut groove, and part of the polyp was grasped by squeezing the snare. Resection was then performed with the use of the electrosurgical current. Repeat snaring and resection were performed in a similar manner to CEPMR. Avulsion, APC, and snare tip coagulation were added in the same manner as CEPMR if they were needed. Fig. 2 shows representative figures of the CEPMR and PEPMR cases. ICC200 and VIO300D (ERBE, Tubingen, Germany) were used as electrosurgical units.

\section{Surveillance Colonoscopy}

All patients were advised to have periodic follow-up sur- veillance colonoscopies or sigmoidoscopies to evaluate local recurrence at the EPMR sites. The first surveillance colonoscopy or sigmoidoscopy was recommended to be at 3 to 12 months after the initial EPMR. The length of time to the second surveillance was determined based on the findings of the first surveillance endoscopy. If the first surveillance showed no recurrence, the second surveillance was recommended to be performed after 1 to 3 years at the discretion of the endoscopist, according to the number, size, and pathology of the polyps resected at the initial colonoscopy with EPMR. If the first surveillance showed local recurrence, endoscopic retreatment was attempted, and the next surveillance endoscopy was recommended again in 3 to 12 months.

\section{Baseline Characteristics and Outcome Measurements}

We investigated the baseline characteristics of the polyps, such as location, size, morphology, and presence or absence of previous biopsy before EPMR. The morphological types of colorectal polyps were classified as the laterally spreading tumor-granular type, the laterally spreading tumor-nongranular type, and the sessile/protruded type. The sessile/protruded type was a $0-$ Is polyp in the Paris classification. One of the complications, intraprocedural bleeding, was defined as bleeding that developed during the endoscopic resection, interrupted the endoscopic resection procedure, and finally required endoscopic hemostasis with the use of hemostatic forceps, APC, and/or clipping. Delayed bleeding was defined as hematochezia and/or melena that developed after the completion of the colonoscopy procedures and required endoscopic hemostasis. Delayed bleeding was divided into early ( $\leq 48$ hours after EPMR) and late ( $>48$ hours after EPMR)
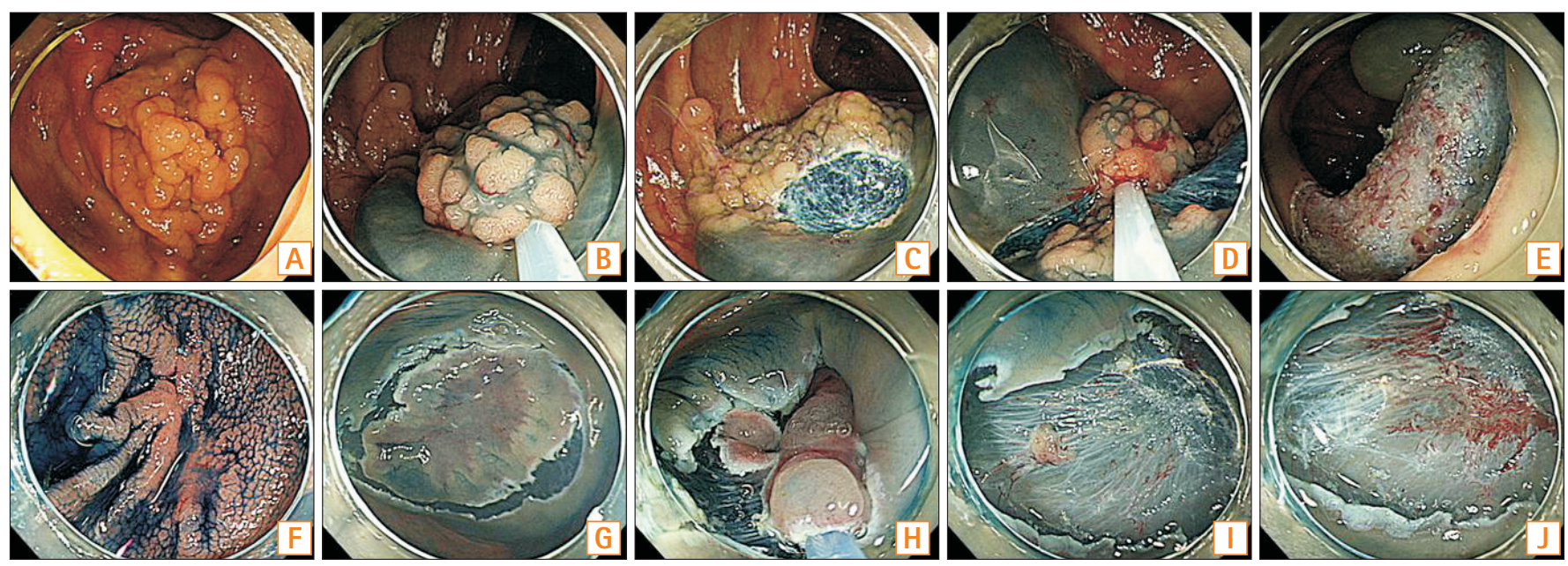

Fig. 2. Conventional endoscopic piecemeal resection (EPMR) (A-E) and precut EPMR (F-J). 
bleeding. Perforation was identified based on the endoscopic evidence of a definite mural defect with the visualization of intraperitoneal organs or periserosal fat tissue.

Resected specimens were retrieved and reconstructed to be as close to the original shape of the polyp as possible. They were then fixed in $10 \%$ formalin and microscopically evaluated. Histological diagnoses were made according to the Vienna classification. ${ }^{15}$ Histological complete resection was defined as the absence of tumor cells at the lateral and deep resection margins of the reconstructed specimen. The definition of a superficial submucosal cancer was a cancer with a depth of submucosal cancer invasion $<1,000 \mu \mathrm{m}$ from the muscularis mucosa.

During surveillance endoscopy, routine biopsy was not performed if the EPMR site showed a completely clear scar without any abnormality. Biopsy was performed if a visible recurrent lesion was detected at the scar or any suspicious abnormality was observed, even if it was subtle. Recurrence was defined as a histologically confirmed colorectal neoplasm that was detected on the scar of the previous EPMR site. Late recurrence was defined as local recurrence discovered at the subsequent colonoscopy approximately 12 months after the first surveillance colonoscopy in which the local recurrence was absent. If a recurrent lesion was detected, endoscopic treatment was attempted. The principal endoscopic treatment method for recurrences was EMR, and supplementary methods, such as cold biopsy, hot biopsy, and APC, were applied if necessary to completely remove all the recurrent lesions.

\section{Statistical Analysis}

Continuous variables were expressed as means with SD or medians with interquartile range (IQR), as appropriate. Continuous parameters were analyzed by the Student $t$-test or the Mann-Whitney $U$-test. Categorical variables were compared by the chi-square test or Fisher exact test, as appropriate. A logistic regression model was used for multivariate analysis of risk factors for recurrence. $P$-values of $<0.05$ were considered to indicate statistical significance. All statistical analyses were performed with SPSS version 21.0 for Windows (IBM Corp., Armonk, NY, USA).

\section{RESULTS}

\section{Baseline Characteristics}

The baseline characteristics of the patients, polyps, and
EPMR procedures are summarized in Table 1. Of 359 lesions in 344 patients, CEPMR was performed for 312 lesions (87\%) and PEPMR was performed for 47 lesions (13\%). The mean age of the patients was similar in the CEPMR and PEPMR groups. The morphological types of the lesions were not different in the 2 groups. However, the mean diameter of the tumors treated with PEPMR $(28.5 \pm 15.4 \mathrm{~mm})$ was larger than that of those treated with CEPMR $(23.7 \pm 12.0 \mathrm{~mm})(P=0.009)$.

Table 1. Baseline Characteristics of Patients, Polyps, and EPMR Procedures

\begin{tabular}{|c|c|c|c|}
\hline & $\begin{array}{l}\text { CEPMR } \\
(n=312)\end{array}$ & $\begin{array}{l}\text { PEPMR } \\
(n=47)\end{array}$ & $P$-value \\
\hline Age (yr) & $63.0 \pm 9.8$ & $62.3 \pm 9.9$ & 0.629 \\
\hline Male sex & $196(62.8)$ & $29(61.7)$ & 0.883 \\
\hline Experienced endoscopists & $296(94.9)$ & $45(95.7)$ & $>0.999$ \\
\hline Location of lesions & & & $<0.001$ \\
\hline Cecum-ascending colon & $175(55.8)$ & $11(23.4)$ & \\
\hline Transverse colon & $52(16.7)$ & $10(21.3)$ & \\
\hline $\begin{array}{l}\text { Descending colon-sigmoid } \\
\text { colon }\end{array}$ & $53(17.0)$ & $12(25.5)$ & \\
\hline Rectum & $33(10.6)$ & $14(29.8)$ & \\
\hline Morphology of lesions & & & 0.642 \\
\hline LST-G & $127(40.7)$ & $21(44.7)$ & \\
\hline LST-NG & $135(43.3)$ & $17(36.2)$ & \\
\hline Sessile/protruded & $50(16.0)$ & $9(19.1)$ & \\
\hline Prior biopsy & & & 0.075 \\
\hline No & $75(24.0)$ & $6(12.8)$ & \\
\hline Yes & $231(74.0)$ & $38(80.9)$ & \\
\hline Unknown & $5(1.6)$ & $3(6.4)$ & \\
\hline Lesion size $(\mathrm{mm})$ & $23.7 \pm 12.0$ & $28.5 \pm 15.4$ & 0.009 \\
\hline Non-lifting sign & $50(16.0)$ & $10(21.7)$ & 0.333 \\
\hline $\begin{array}{l}\text { Supplementary procedures after } \\
\text { EPMR }\end{array}$ & & & 0.310 \\
\hline None & $94(30.1)$ & $21(44.7)$ & \\
\hline Avulsion by hot biopsy forceps & $26(8.3)$ & $4(8.5)$ & \\
\hline APC & $153(49.0)$ & $19(40.4)$ & \\
\hline Avulsion+APC & $3(1.0)$ & 0 & \\
\hline Snare tip coagulation & $36(11.5)$ & $3(6.4)$ & \\
\hline
\end{tabular}




\section{Short-Term Outcomes after EPMR}

The details of the procedure-related variables and histological outcomes are described in Table 2 . The average number of resected pieces per case was similar in the CEPMR and PEPMR groups. The distribution of histological diagnoses was also similar in both groups. Adenoma was the most common pathology in both groups. The histological complete resection rate was higher in the CEPMR group (40.1\%) than in the PEPMR group (31.9\%) $(P=0.003)$. Intraprocedural bleeding was more frequent in the PEPMR group than in the CEPMR group ( $31.9 \%$ vs. $12.5 \%, P=0.001$ ).

\section{Long-Term Outcomes after EPMR}

The median time to the first surveillance colonoscopy was 7.9 months (IQR, 4.9-12.8 months), and the overall duration of follow-up was 20.0 months (IQR, 12.3-38.3 months). The overall recurrence rate during the study period was
$7.5 \%(27 / 359)$. The recurrence rates were not significantly different in the CEPMR and PEPMR groups (7.7\% vs. $6.4 \%$, $P>0.751$ ) (Table 2). Local recurrence at the first surveillance endoscopy was observed in 18 of 312 lesions (5.8\%) after CEPMR and in 3 of 47 lesions (6.4\%) after PEPMR. Among lesions that did not have a recurrence at the first surveillance colonoscopy, the rate of late recurrence on subsequent surveillance colonoscopy was 3.9\% (6/152) after CEPMR and $0 \%$ after PEPMR (Fig. 1). The median time to late recurrence after CEPMR was 16.0 months (IQR, 14.5-20.6 months). Recurrence rates increased as lesion size increased in both groups (Table 3).

In the univariate analysis, local recurrence was associated with tumor size, tumor morphology, number of resected pieces, and perforation. However, multivariate analysis revealed that larger tumor size was the only independent risk factor for recurrence (Table 4).

Table 2. Clinical Outcomes after EPMR

\begin{tabular}{|c|c|c|c|}
\hline & CEPMR $(n=312)$ & PEPMR $(n=47)$ & $P$-value \\
\hline No. of resected pieces & $3.4 \pm 2.3$ & $3.2 \pm 1.7$ & 0.903 \\
\hline Histology & & & 0.072 \\
\hline Adenoma/low grade dysplasia & $173(55.4)$ & $23(48.9)$ & \\
\hline Adenoma/high grade dysplasia & $63(20.2)$ & $14(29.8)$ & \\
\hline Sessile serrated adenoma/polyp & $28(9.0)$ & 0 & \\
\hline Mucosal cancer & $33(10.6)$ & 7 (14.9) & \\
\hline Superficial submucosal cancer & $6(1.9)$ & $3(6.4)$ & \\
\hline Deep submucosal cancer ${ }^{a}$ & $1(0.3)$ & 0 & \\
\hline Others & $8(2.6)$ & 0 & \\
\hline Histological complete resection & $125(40.1)$ & 15 (31.9) & 0.003 \\
\hline \multicolumn{4}{|l|}{ Complication } \\
\hline Perforation & $3(1.0)$ & $1(2.1)$ & 0.431 \\
\hline Intraprocedural bleeding & $39(12.5)$ & 15 (31.9) & 0.001 \\
\hline \multicolumn{4}{|l|}{ Delayed bleeding } \\
\hline Early delayed bleeding ( $\leq 48 \mathrm{hr}$ ) & $9(2.9)$ & $3(6.4)$ & 0.198 \\
\hline Late delayed bleeding (>48 hr) & $3(1.0)$ & $1(2.1)$ & 0.431 \\
\hline Follow-up duration (mo) & $20.5(12.3-38.2)$ & $18.2(12.1-43.0)$ & 0.696 \\
\hline No. of surveillance endoscopies & $2.0 \pm 1.2$ & $2.1 \pm 1.1$ & 0.258 \\
\hline Overall recurrence & $24(7.7)$ & $3(6.4)$ & 0.751 \\
\hline
\end{tabular}

Values are presented as mean $\pm S D$, number (\%), or median (interquartile range).

${ }^{a}$ The patient refused additional surgery because of existing comorbidities of liver cirrhosis and lymphoma. She was followed up for 12 months without recurrence, but died of septic shock as a complication of lymphoma treatment after 3 years.

EPMR, endoscopic piecemeal mucosal resection; CEPMR, conventional EPMR; PEPMR, precut EPMR. 
Table 3. Recurrence Rates According to Lesion Size

\begin{tabular}{|c|c|c|c|c|}
\hline Recurrence rate & CEPMR $(n=312)$ & PEPMR $(n=47)$ & Total $(n=359)$ & $P$-value \\
\hline Overall recurrence & $7.7(24 / 312)$ & $6.4(3 / 47)$ & $7.5(27 / 359)$ & $>0.999$ \\
\hline Recurrence in polyps $\geq 20 \mathrm{~mm}$ & $10.6(21 / 199)$ & $7.9(3 / 38)$ & $10.1(24 / 237)$ & 0.775 \\
\hline $10-19$ & $2.7(3 / 113)$ & $0(0 / 9)$ & $2.5(3 / 122)$ & $>0.999$ \\
\hline $20-29$ & $7.4(8 / 108)$ & $12.5(2 / 16)$ & $8.1(10 / 124)$ & 0.626 \\
\hline$\geq 40$ & $18.2(6 / 33)$ & $11.1(1 / 9)$ & $16.7(7 / 42)$ & $>0.999$ \\
\hline$P$-value & 0.001 & 0.727 & 0.002 & \\
\hline
\end{tabular}

Values are presented as percent (number/total number).

CEPMR, conventional endoscopic piecemeal mucosal resection; PEPMR, precut EPMR.

Table 4. Univariate and Multivariate Analyses of Risk Factors for Local Recurrence

\begin{tabular}{|c|c|c|c|c|}
\hline & \multicolumn{2}{|c|}{ Univariate analysis } & \multicolumn{2}{|c|}{ Multivariate analysis } \\
\hline & OR $(95 \% \mathrm{Cl})$ & $P$-value & OR $(95 \% \mathrm{Cl})$ & $P$-value \\
\hline Size of lesion (mm) & & 0.016 & & 0.015 \\
\hline$<20$ & 1 (reference) & & 1 (reference) & \\
\hline 20-39 & 3.79 (1.09-13.21) & & 3.79 (1.09-13.21) & 0.037 \\
\hline$\geq 40$ & 7.93 (1.95-32.30) & & 7.93 (1.95-32.30) & $<0.001$ \\
\hline Morphology of lesion & & 0.005 & & \\
\hline Sessile/protruded & 1 (reference) & & & \\
\hline LST-NG & $1.58(0.36-6.81)$ & & & \\
\hline LST-G & 4.33 (1.57-11.93) & & & \\
\hline No. of pieces & & 0.019 & & \\
\hline$<4$ & 1 (reference) & & & \\
\hline$\geq 4$ & $2.58(1.17-5.69)$ & & & \\
\hline Perforation & & 0.032 & & \\
\hline No & 1 (reference) & & & \\
\hline Yes & 4.47 (1.14-17.67) & & & \\
\hline
\end{tabular}

LST-NG, laterally spreading tumor-nongranular type; LST-G, LST-granular type.

\section{Performance of Endoscopic Treatment for Recurrent Lesions}

Among 27 recurrent tumors, 2 cases recurred as sessile serrated adenomas and the remaining 25 cases recurred as adenomas/villous adenomas. No case recurred as cancer after removal of an adenoma. Salvage endoscopic treatment was performed for all 21 recurrent lesions at the first surveillance endoscopy. Single endoscopic treatment methods, such as cold forceps polypectomy or EMR, were used in 3 recurrent lesions, and combination methods (EMR plus APC, EMR plus hot biopsy, EMR plus hot biopsy plus APC, cold forceps polypectomy plus APC, or hot biopsy plus APC) were used in 18 recurrent lesions. Of these 21 recurrent lesions, 15 underwent surveillance endoscopies after endoscopic treatment. Of the latter 15 lesions, 3 (20.0\%) had a second recurrence at the subsequent surveillance endoscopy; 1 patient underwent surgical appendectomy because the recurrence was at the appendiceal orifice, and the other 2 underwent repeat endoscopic treatment.

Salvage endoscopic treatment was performed for all 6 late recurrences at the second or third surveillance endoscopy in cases without recurrence at the first surveillance endoscopy. A single endoscopic treatment modality was used in 5 late 
recurrences and a combination method in 1 late recurrence. Of these 6 patients with late recurrence, 5 underwent subsequent surveillance endoscopy and showed no further recurrence.

In summary, 21 lesions (5.8\%) showed local recurrence at the first surveillance endoscopy, and 6 lesions (3.9\%) showed late recurrence at subsequent surveillances, although they had not had recurrence at the first surveillance. All 27 recurrences were treated endoscopically. A single modality was used in 8 recurrences (29.6\%), and combination methods were used in 19 recurrences (70.4\%) (Table 5). Twenty of 27 recurrences were followed up by subsequent endoscopy. Of these, 1 (5.0\%) was treated by surgical appendectomy because of re-recurrence at the appendiceal orifice, and the other 19 (95.0\%) were cured by endoscopic treatment, although 3 of the 19 showed second or third recurrences, which were treated by repeat endoscopic treatment. A flowchart detailing recurrence and subsequent endoscopic treatment is presented in Fig. 1.

\section{DISCUSSION}

In our meticulous review, we found that the rates of recurrence after CEPMR and PEPMR were not different. Large tumor size increased the risk of local recurrence after EPMR. Recurrent lesions could be managed by endoscopic treatment in most cases, which was usually combination therapy rather than a single method.

Table 5. Endoscopic Treatment Methods for the First Recurrent Lesions ${ }^{\mathrm{a}}$ after EPMR

\begin{tabular}{cc}
\hline Treatment modalities & No. of cases (\%) \\
\hline Single modality & $8(29.6)$ \\
Cold forceps polypectomy & $5(18.5)$ \\
EMR & $3(11.1)$ \\
Combination modality & $19(70.4)$ \\
Cold forceps polypectomy+APC & $6(22.2)$ \\
Hot biopsy+APC & $2(7.4)$ \\
EMR+APC & $7(25.9)$ \\
EMR+hot biopsy & $3(11.1)$ \\
EMR+hot biopsy+APC & $1(3.7)$ \\
\hline
\end{tabular}

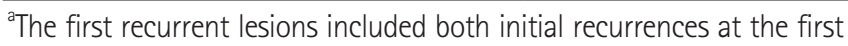
surveillance endoscopy $(n=21)$ and late recurrences at the second and third surveillance endoscopies without recurrence at the first surveillance endoscopy $(n=6)$.

EPMR, endoscopic piecemeal mucosal resection; EMR, endoscopic mucosal resection; $\mathrm{APC}$, argon plasma coagulation.
Previous studies reported that the local recurrence rate after EPMR was $6.9 \%$ to $23.5 \%{ }^{1,3,16-19}$ A recent meta-analysis showed that the recurrence rate after EPMR was $11 \%$ to $54 \%$ for lesions $\geq 20 \mathrm{~mm}$ and the pooled estimate of recurrence risk was $22 \%$, compared with only $3 \%$ after en bloc EMR. ${ }^{18}$ In our study, the overall local recurrence rates of CEPMR and PEPMR were 7.7\% (24/312) and 6.4\% (3/47) for colorectal polyps $\geq 10 \mathrm{~mm}(P>0.999)$. For polyps $\geq 20 \mathrm{~mm}$, the recurrence rates were $10.6 \%(21 / 199)$ after CEPMR and $7.9 \%$ (3/38) after PEPMR $(P=0.775)$. The recurrence rates in our study are within the ranges of earlier reports. The rate of late recurrence at the second or third surveillance endoscopy without recurrence at the first surveillance was $3.9 \%(6 / 152)$ after CEPMR, whereas there was no late recurrence after PEPMR. The late recurrence rate after CEPMR in our study is also consistent with a previous study that showed a late recurrence rate of approximately $4 \% .{ }^{1,2}$ An interesting point of our study is that there was no difference in recurrence rates after CEPMR and PEPMR. Although precut incision with a safety margin can guarantee a clear lateral resection margin and theoretically provide a higher complete resection rate than conventional EMR, ${ }^{11,12}$ it cannot eliminate the risk of tumor remaining between the pieces after EPMR. The tumor remaining between the pieces may grow and lead to recurrent tumors detected at follow-up surveillance endoscopy. We suggest this may be one reason that the recurrence rates were not different between CEPMR and PEPMR. Another point worthy of note in our study is the lower histological complete resection rate (31.9\%) after PEPMR compared with $40.1 \%$ after CEPMR. We expected a higher histological complete resection rate after PEPMR because of the precut incision with safety margin. However, the result was contrary to our hypothesis. We believe that the lower complete resection rate after PEPMR may be related to thermal injury of the resection margin during circumferential incision. ${ }^{11}$ Although the resection itself was complete, without involvement of the resection margins by tumor tissue, thermal injury may have made precise histological evaluation difficult and resulted in positive or indeterminate resection margins.

Larger tumor size ( $\geq 40 \mathrm{~mm}$ ) was the only independent risk factor for local recurrence after EPMR, regardless of piecemeal resection methods. As the size of the polyp increases, the number of pieces may increase during EPMR. If the number of pieces increases, the risk of remnant tumor between pieces may increase, thereby increasing the risk of recurrence. Therefore, we suggest that adequate strategies to decrease the risk of recurrence should be considered after EPMR of large polyps, especially those of diameter $\geq 40 \mathrm{~mm}$. 
Utilizing APC may be 1 option to decrease the risk of recurrence, ${ }^{20,21}$ and routine application of APC may be considered after EPMR of polyps with $\geq 40 \mathrm{~mm}$ diameter. Avulsion of visible residual tumor with hot biopsy forceps may be another method to minimize the risk of recurrence after EPMR. ${ }^{22}$ In addition, we suggest that more careful endoscopic surveillance should be considered after EPMR of polyps with $\geq 40$ $\mathrm{mm}$ diameter because of the higher risk of recurrence.

Although local recurrence rates were not low, endoscopic treatment ultimately provided a high cure rate of $95 \%$ (19/20), despite repeat recurrences in some cases. Only 1 case $(5 \%)$ underwent surgical management because the recurrent lesion involved the appendiceal orifice and extended into the appendiceal lumen. Regarding the endoscopic treatment methods, $70.4 \%$ of all recurrences (19/27) were treated not by single endoscopic therapy but by combination therapy (EMR plus APC, EMR plus hot biopsy, EMR plus hot biopsy plus APC, cold forceps polypectomy plus APC, or hot biopsy plus APC). Because recurrent lesions after endoscopic resection, especially wide resection, such as EPMR of large polyps, may develop in the background of the scar tissue, the non-lifting sign may be present and endoscopic resection may be much more difficult than endoscopic resection of polyps with no previous attempt at resection. ${ }^{21,23}$ Hence, a single endoscopic treatment method may not be sufficient for complete removal of the recurrent lesions on the scar. ${ }^{24}$ This may be a reason why combination therapy was used in most cases of recurrence. Even though a combination of methods was needed, endoscopic management was very effective for treatment of recurrent lesions after EPMR. Future studies should investigate the most useful endoscopic methods for treatment of recurrent lesions on the scar.

Complication rates after CEPMR and PEPMR were not different except for a higher rate of intraprocedural bleeding after PEPMR. Circumferential precutting by the snare tip or endo-knives may have been the reason for higher intraprocedural bleeding during PEPMR. Despite the absence of differences in delayed bleeding and other complications, considering the more frequent intraprocedural bleeding during PEPMR and the absence of difference in local recurrence rates between CEPMR and PEPMR, we suggest that PEPMR may not be recommended routinely for resection of large polyps and CEPMR should be the first choice. However, because of the theoretical advantage of minimal risk of slipping of the tumor out of the snare during closure of the snare, PEPMR may be indicated for resection of very flat tumors that are liable to slip out of the snare. Our study was a retrospective one, and we could not evaluate and analyze the usefulness of PEPMR from this viewpoint. Future studies are needed to clearly define the possible indications for PEPMR.

Our study had several limitations. First, because it was a retrospective study based on experience in a single center, selection bias related to the choice of PEPMR might have influenced the clinical outcomes and recurrence rates. Additionally, we searched for EPMR cases from the database of our institution by the computerized searching system using the term "piecemeal." Because the searching system was not perfect, many EPMR cases may have been missed, which is another cause of selection bias of this study. The second limitation is the absence of a detailed analysis on the probable difference in the endoscopic skill between experienced and trainee endoscopists. Therefore, we do not know whether the expertise in endoscopic technique may have influenced the outcomes after EPMR. Third, because only half of the enrolled patients underwent a second surveillance, the local recurrence rates might be underestimated. Finally, the median follow-up period was $<2$ years. Despite these weaknesses, we believe that the present study is meaningful because, to the best of our knowledge, our investigation is the first study comparing clinical outcomes between CEPMR and PEPMR.

In conclusion, local recurrence rates were not different in CEPMR and PEPMR. Large tumor size was the only independent risk factor for local recurrence after EPMR. Endoscopic treatment for recurrent lesions resulted in a high cure rate, although the majority of recurrences required a combination of 2 or more endoscopic treatment methods.

\section{REFERENCES}

1. Moss A, Williams SJ, Hourigan LF, et al. Long-term adenoma recurrence following wide-field endoscopic mucosal resection (WF-EMR) for advanced colonic mucosal neoplasia is infrequent: results and risk factors in 1000 cases from the Australian Colonic EMR (ACE) study. Gut 2015;64:57-65.

2. Khashab M, Eid E, Rusche M, Rex DK. Incidence and predictors of "late" recurrences after endoscopic piecemeal resection of large sessile adenomas. Gastrointest Endosc 2009;70:344-349.

3. Oka S, Tanaka S, Saito Y, et al. Local recurrence after endoscopic resection for large colorectal neoplasia: a multicenter prospective study in Japan. Am J Gastroenterol 2015;110:697-707.

4. Tanaka S, Haruma K, Oka S, et al. Clinicopathologic features and endoscopic treatment of superficially spreading colorectal neoplasms larger than 20 mm. Gastrointest Endosc 2001;54:6266. 
5. Yang DH, Jeong GH, Song Y, et al. The feasibility of performing colorectal endoscopic submucosal dissection without previous experience in performing gastric endoscopic submucosal dissection. Dig Dis Sci 2015;60:3431-3441.

6. Woodward TA, Heckman MG, Cleveland P, De Melo S, Raimondo M, Wallace M. Predictors of complete endoscopic mucosal resection of flat and depressed gastrointestinal neoplasia of the colon. Am J Gastroenterol 2012;107:650-654.

7. Saito Y, Fukuzawa M, Matsuda T, et al. Clinical outcome of endoscopic submucosal dissection versus endoscopic mucosal resection of large colorectal tumors as determined by curative resection. Surg Endosc 2010;24:343-352.

8. Lee EJ, Lee JB, Lee SH, Youk EG. Endoscopic treatment of large colorectal tumors: comparison of endoscopic mucosal resection, endoscopic mucosal resection-precutting, and endoscopic submucosal dissection. Surg Endosc 2012;26:2220-2230.

9. Tajika M, Niwa Y, Bhatia V, et al. Comparison of endoscopic submucosal dissection and endoscopic mucosal resection for large colorectal tumors. Eur J Gastroenterol Hepatol 2011;23:10421049 .

10. Byeon JS, Yang DH, Kim KJ, et al. Endoscopic submucosal dissection with or without snaring for colorectal neoplasms. Gastrointest Endosc 2011;74:1075-1083.

11. Hong YM, Kim HW, Park SB, Choi CW, Kang DH. Endoscopic mucosal resection with circumferential incision for the treatment of large sessile polyps and laterally spreading tumors of the colorectum. Clin Endosc 2015;48:52-58.

12. Sakamoto T, Matsuda T, Nakajima T, Saito Y. Efficacy of endoscopic mucosal resection with circumferential incision for patients with large colorectal tumors. Clin Gastroenterol Hepatol 2012;10:22-26.

13. Kim HG, Sethi S, Banerjee S, Friedland S. Outcomes of endoscopic treatment of second recurrences of large nonpedunculated colorectal adenomas. Surg Endosc 2016;30:2457-2464.

14. Sakamoto T, Saito Y, Matsuda T, Fukunaga S, Nakajima T, Fujii T. Treatment strategy for recurrent or residual colorectal tumors after endoscopic resection. Surg Endosc 2011;25:255-260.
15. Schlemper RJ, Riddell RH, Kato Y, et al. The Vienna classification of gastrointestinal epithelial neoplasia. Gut 2000;47:251255.

16. Seo GJ, Sohn DK, Han KS, et al. Recurrence after endoscopic piecemeal mucosal resection for large sessile colorectal polyps. World J Gastroenterol 2010;16:2806-2811.

17. Hotta K, Fujii T, Saito Y, Matsuda T. Local recurrence after endoscopic resection of colorectal tumors. Int J Colorectal Dis 2009;24:225-230.

18. Belderbos TD, Leenders M, Moons LM, Siersema PD. Local recurrence after endoscopic mucosal resection of nonpedunculated colorectal lesions: systematic review and meta-analysis. Endoscopy 2014;46:388-402.

19. Sakamoto T, Matsuda T, Otake Y, Nakajima T, Saito Y. Predictive factors of local recurrence after endoscopic piecemeal mucosal resection. J Gastroenterol 2012;47:635-640.

20. Regula J, Wronska E, Polkowski M, et al. Argon plasma coagulation after piecemeal polypectomy of sessile colorectal adenomas: long-term follow-up study. Endoscopy 2003;35:212-218.

21. Tsiamoulos ZP, Bourikas LA, Saunders BP. Endoscopic mucosal ablation: a new argon plasma coagulation/injection technique to assist complete resection of recurrent, fibrotic colon polyps (with video). Gastrointest Endosc 2012;75:400-404.

22. Holmes I, Kim HG, Yang DH, Friedland S. Avulsion is superior to argon plasma coagulation for treatment of visible residual neoplasia during EMR of colorectal polyps (with videos). Gastrointest Endosc 2016;84:822-829.

23. Kim HG, Thosani N, Banerjee S, Chen A, Friedland S. Underwater endoscopic mucosal resection for recurrences after previous piecemeal resection of colorectal polyps (with video). Gastrointest Endosc 2014;80:1094-1102.

24. Kim EK, Han DS, Ro Y, Eun CS, Yoo KS, Oh YH. The submucosal fibrosis: what does it mean for colorectal endoscopic submucosal dissection? Intest Res 2016;14:358-364. 